\title{
RESEARCH
}

Open Access

\section{Brush swab as a noninvasive surrogate for tissue biopsies in epigenomic profiling of oral cancer}

Chi T. Viet ${ }^{1 *}$ (D), Xinyu Zhang ${ }^{2,3}, K^{2} X^{2,3}$, Gary Yu ${ }^{4}$, Kesava Asam ${ }^{5,6}$, Carissa M. Thomas ${ }^{7}$, Nicholas F. Callahan ${ }^{8}$, Coleen Doan ${ }^{1}$, Paul C. Walker ${ }^{9}$, Khanh Nguyen ${ }^{9}$, Stephanie C. Kidd ${ }^{9}$, Steve C. Lee ${ }^{9}$, Anupama Grandhi', Clint T. Allen ${ }^{10}$, Simon Young ${ }^{11}$, James C. Melville ${ }^{11}$, Jonathan W. Shum ${ }^{11}$, Dan T. Viet ${ }^{12}$, Alan S. Herford ${ }^{1}$, Dylan F. Roden ${ }^{13}$, Manuel L. Gonzalez ${ }^{14}$, Jiang F. Zhong ${ }^{15}$ and Bradley E. Aouizerat ${ }^{2,3,4}$

\begin{abstract}
Background: Oral squamous cell carcinoma (OSCC) has poor survival rates. There is a pressing need to develop more precise risk assessment methods to tailor clinical treatment. Epigenome-wide association studies in OSCC have not produced a viable biomarker. These studies have relied on methylation array platforms, which are limited in their ability to profile the methylome. In this study, we use MethylCap-Seq (MC-Seq), a comprehensive methylation quantification technique, and brush swab samples, to develop a noninvasive, readily translatable approach to profile the methylome in OSCC patients.

Methods: Three OSCC patients underwent collection of cancer and contralateral normal tissue and brush swab biopsies, totaling 4 samples for each patient. Epigenome-wide DNA methylation quantification was performed using the SureSelectXT Methyl-Seq platform. DNA quality and methylation site resolution were compared between brush swab and tissue samples. Correlation and methylation value difference were determined for brush swabs vs. tissues for each respective patient and site (i.e., cancer or normal). Correlations were calculated between cancer and normal tissues and brush swab samples for each patient to determine the robustness of DNA methylation marks using brush swabs in clinical biomarker studies.

Results: There were no significant differences in DNA yield between tissue and brush swab samples. Mapping efficiency exceeded $90 \%$ across all samples, with no differences between tissue and brush swabs. The average number of CpG sites with at least 10x depth of coverage was 2,716,674 for brush swabs and 2,903,261 for tissues. Matched tissue and brush swabs had excellent correlation ( $r=0.913$ for cancer samples and $r=0.951$ for normal samples). The methylation profile of the top $1000 \mathrm{CpGs}$ was significantly different between cancer and normal samples (mean $p$-value $=0.00021$ ) but not different between tissues and brush swabs (mean $p$-value $=0.11$ ).
\end{abstract}

\footnotetext{
*Correspondence: cviet@llu.edu

'Department of Oral and Maxillofacial Surgery, Loma Linda University School of Dentistry, Loma Linda, CA, USA

Full list of author information is available at the end of the article
}

(c) The Author(s). 2021 Open Access This article is licensed under a Creative Commons Attribution 4.0 International License, which permits use, sharing, adaptation, distribution and reproduction in any medium or format, as long as you give appropriate credit to the original author(s) and the source, provide a link to the Creative Commons licence, and indicate if changes were made. The images or other third party material in this article are included in the article's Creative Commons licence, unless indicated otherwise in a credit line to the material. If material is not included in the article's Creative Commons licence and your intended use is not permitted by statutory regulation or exceeds the permitted use, you will need to obtain permission directly from the copyright holder. To view a copy of this licence, visit http://creativecommons.org/licenses/by/4.0/ The Creative Commons Public Domain Dedication waiver (http://creativecommons.org/publicdomain/zero/1.0/) applies to the data made available in this article, unless otherwise stated in a credit line to the data. 
Conclusions: Our results demonstrate that MC-Seq is an efficient platform for epigenome profiling in cancer biomarker studies, with broader methylome coverage than array-based platforms. Brush swab biopsy provides adequate DNA yield for MC-Seq, and taken together, our findings set the stage for development of a non-invasive methylome quantification technique for oral cancer with high translational potential.

Keywords: Methylation biomarker, Oral cancer, Head and neck cancer, Epigenetic biomarker, Brush swab, Brush biopsy, Biomarker, MethylCap-Seq, Methylation array

\section{Introduction}

Each year 30,000 patients are diagnosed with oral cavity squamous cell carcinoma (OSCC), and unfortunately the incidence is on the rise [1-3].. Even for these early stage patients, the five-year survival rate is $60 \%$ [4]. Poor survival rates are in part due to inaccurate risk prediction. Early stage OSCC is primarily treated with surgical resection of the cancer, with or without adjuvant treatments such as an elective lymphadenectomy, radiation, or chemoradiation, for patients with high risk features. Currently, risk prediction to assign adjuvant treatment is entirely based on clinicopathologic information. Multiple retrospective and prospective studies have shown that these standard clinicopathologic factors have moderate accuracy with a concordance statistic (c-statistic) of 0.7 $[4,5]$. The key to improving survival in OSCC lies in developing more accurate risk prediction methods, particularly in early stage patients. Although OSCC is a heavily epigenetically-regulated cancer [6], optimizing risk prediction using methylation features remains in its infancy. Methylation is one of the most frequent epigenetic changes in early oral carcinogenesis that is linked to cancer progression [6]. While several methylation studies in OSCC patients [6-17], including our own studies [7, 8], have highlighted differential methylation features between low and high risk patients, none of these studies have resulted in a clinically meaningful biomarker. Two main shortcomings of these previous studies are: 1) failure to use a clinically translatable array platform, and 2) failure to quantify methylation in real time, as cancer treatment is occurring.

With respect to the first challenge, the vast majority of methylation array studies in OSCC have used arraybased platforms. While the Illumina Methylation $450 \mathrm{~K}$ or EPIC array are the most commonly used platforms for epigenome-wide association studies (EWAS), CpG site quantification is restricted at an upper limit of 870,000 sites, and results from these platforms have not been converted into a clinically-accessible risk prediction tool. Furthermore, the EPIC array content is frequently updated to enrich for cancer-associated genes, making comparison across cohorts challenging. Methylation capture sequencing (MC-seq) has a scalable workflow that can quantify methylation in a small subset of genes or the entire genome using next generation sequencing
(NGS), with a higher likelihood of clinical translation due to broader CpG coverage in a more agnostic manner while maintaining its resolution in samples with modest DNA quantities [18].

With respect to the second challenge, clinical translation of a biomarker requires measurement at the onset of treatment in order to determine risk and the need for treatment escalation. Waiting until after cancer removal for the formalin-fixed, paraffin-embedded (FFPE) tissues would limit clinical translatability. The oral cavity has the advantage of being readily accessible for sampling, not only with tissue biopsies, but also with noninvasive techniques. Herein, we determine methylation features using noninvasive brush swabs. Brush swab biopsies of oral cancer and high grade lesions have been used in methylation analysis of a limited number of genes [19-21]. While the search for putative biomarkers is ongoing, this study focuses on the technological aspects of epigenome-wide profiling using noninvasive brush swab samples.

In this study, we hypothesize that brush swab biopsies serve as a robust noninvasive method to quantify cancer-specific methylation features. Using tissue and brush swab biopsies collected from OSCC patients at the time of surgery: 1) we determine the concordance between the methylation signature of cancer tissues and swabs vs. matched normal tissues and swabs using MCSeq, and 2) we establish a workflow in which brush swabs and MC-seq are used at the time of diagnosis to establish a methylation signature that can be used to determine risk of mortality.

\section{Methods}

\section{Patient selection and data collection}

The patients were enrolled in a multi-institutional prospective clinical study in which biological samples and clinicopathologic information were collected. Collection of clinical data and samples was approved by the Institutional Review Board at each institution, which included Loma Linda University (LLU), University of Illinois Chicago (UIC), and University of Alabama at Birmingham (UAB). Patients were eligible if they were $\geq 18$ years of age, had biopsy-proven squamous cell carcinoma of oral cavity sub-sites, including oral tongue, maxillary and mandibular gingiva, hard palate, floor of mouth, buccal mucosa, and lip mucosa, and no previous treatment of 
OSCC. Clinical and pathologic stages were recorded based on the American Joint Committee on Cancer (AJCC) Eighth Edition Staging Manual [22]. We collected the following information from the chart review: age, sex, race, smoking and alcohol use, staging, tumor location, pathologic characteristics, and treatment modalities received in addition to tumor ablation. Biological samples collected at the time of surgery include flashfrozen cancer and contralateral normal tissue, and brush swab biopsies of the cancer and contralateral normal site. Isohelix brush swabs (Boca Scientific) were brushed for a total of 20 times, with 10 times on each surface of the swab, at either the cancer or contralateral normal site. The brush swabs were preserved using 500ul BuccalFix $^{\text {Ta }}$ stabilization solution (Boca Scientific). Samples were stored in $-80^{\circ} \mathrm{C}$. A total of 3 patients were randomly chosen from the ongoing prospective clinical study for the current study.

\section{Nucleic acid extraction and sample preparation}

DNA was extracted from the flash-frozen tissue and brush swabs of the cancer and contralateral normal side of 3 patients, totaling 12 samples (4 samples per patient). Genomic DNA quality was determined by spectrophotometry and concentration was determined by fluorometry. DNA integrity and fragment size were determined using a microfluidic chip run on an Agilent Bioanalyzer.

\section{MC-seq target enrichment library prep}

Indexed paired-end whole-genome sequencing libraries were prepared using the SureSelect XT Methyl-Seq kit (Agilent). Genomic DNA was sheared to a fragment length of $150-200 \mathrm{bp}$ using the Covaris E220 system. Fragmented sample size distribution was determined using the Caliper LabChip GX system (PerkinElmer). Fragmented DNA ends were repaired with T4 DNA Polymerase and Polynucleotide Kinase and "A" base was added using Klenow fragment followed by AMPure XP bead-based purification (Beckman Coulter). The methylated adapters were ligated using T4 DNA ligase followed by bead purification with AMPure XP. Quality and quantity of adapter-ligated DNA were assessed with the Caliper LabChip GX system. Samples were enriched for targeted methylation sites by using the custom SureSelect Methyl-Seq Capture Library. Hybridization was performed at $65^{\circ} \mathrm{C}$ for $16 \mathrm{~h}$ using a thermal cycler. Once the enrichment was completed, the samples were mixed with streptavidin-coated beads (Thermo Fisher Scientific) and washed with a series of buffers to remove nonspecific DNA fragments. DNA fragments were eluted from beads with $0.1 \mathrm{M} \mathrm{NaOH}$. Unmethylated $\mathrm{C}$ residues of enriched DNA underwent bisulfite conversion using the EZ DNA Methylation-Gold Kit (Zymo Research). The SureSelect enriched and bisulfite-converted libraries underwent PCR amplification using custom made primers (IDT). Dual-indexed libraries were quantified by quantitative polymerase chain reaction (qPCR) with the Library Quantification Kit (KAPA Biosystems) and inserts size distribution was assessed using the Caliper LabChip GX system.

\section{Flow cell preparation and sequencing}

Samples were sequenced using $100 \mathrm{bp}$ paired-end sequencing on an Illumina HiSeq NovaSeq according to Illumina protocol. A positive control (prepared bacteriophage Phi X library) was added into every lane at a concentration of $0.3 \%$ to assess sequencing quality in real time.

\section{Preprocessing and quality control}

Signal intensities were converted to individual base calls during each run using the system's Real Time Analysis software. Sample de-multiplexing was performed using Illumina's CASAVA 1.8.2 software suite. The sample error rate was required to be less than $1 \%$ and the distribution of reads per sample in a lane to be within reasonable tolerance. Sequence data quality were examined using FastQC (ver. 0.11.8). Adapter sequences and fragments with poor quality were removed by Trim_galore (ver. 0.6.3_dev). Bismark pipelines (ver. v0.22.1_dev) were used to align the reads to the bisulfite human genome (hg19) with default parameters [23]. Sample alignment to the human genome was performed using bowtie 2 (ver. 2.3.5.1). Quality-trimmed paired-end reads were converted into a bisulfite forward ( $\mathrm{C}->\mathrm{T}$ conversion) or reverse (G->A conversion) strand read. Duplicated reads were removed from the Bismark mapping output and $\mathrm{CpG}$ extracted. All CpG sites were grouped by sequencing coverage (i.e., read depth); $\mathrm{CpG}$ sites with coverage $\geq 10 x$ depth were retained for analysis to ensure high MC-Seq data quality. Genes were annotated using Homer annotatePeaks.pl. With this software, the promoter region is defined as 1 kilobase from the transcription start site (TSS).

\section{Comparison of methylation between tissue and brush swab biopsies}

Benjamini-Hochberg FDR was applied to adjust $p$ values per CpG site. Pearson correlations were calculated between tissue and brush biopsy samples of matched anatomic sites, and cancer and normal samples from the same patients. Pearson correlation and absolute difference were calculated among common CpG sites between the samples. Scatterplots were rendered showing the correlation of $\beta$ values from all CpG sites measured by $\mathrm{MC}$-seq. Separate scatterplots were rendered showing the concordance of these CpG sites between tissues and brush swabs for the cancer sites and the normal sites. 
Student $\mathrm{t}$-tests were performed to compare $\beta$ values between cancer and normal groups or tissue and brush swab groups. The most significant $1000 \mathrm{CpGs}$ features in cancer vs. normal groups were selected. Based on these results the $-\log 10$ (t-test $p$-value) was calculated for each of the $1000 \mathrm{CpG}$ sites to compare the degree of divergence in the significance of the test statistics for these 1000 CpG between 1) cancer vs. normal and 2) tissue vs. brush swabs.

\section{Statistical analyses}

Statistical analyses were performed in $\mathrm{R}$ environment (v. 4.1.0).

\section{Results}

\section{Patient cohort characteristics and DNA quality}

Clinicopathologic information for the 3 enrolled patients are detailed in Table 1. The 3 patients comprised both early and late stage OSCC (stage I and IV), as well as varying tobacco and alcohol consumption habits. Patients were 49 and 68 years old. Two patients were male and one was female. All patients were white, nonHispanic. Cancer and contralateral normal tissue and brush swab biopsies collected at the time of surgery underwent DNA extraction, with the yield and quality shown in Table 2. With a total input volume of $30 \mu \mathrm{L}$ for each sample, total input for tissue DNA ranged from $187 \mathrm{ng}$ to $660 \mathrm{ng}$, and an average of $390 \mathrm{ng}$. Total input for swab DNA ranged from $51 \mathrm{ng}$ to $1998 \mathrm{ng}$, with an average of $532 \mathrm{ng}$. The input range was consistent with our previous study demonstrating reproducible CpG site quantification using MC-Seq across this range [18]. In our previous study, DNA quantity as low as $150-300 \mathrm{ng}$ and DNA quality comparable to the findings in Table 2 were successfully amplified using our workflow.

\section{MC-Seq mapping efficiency assessment}

Table 3 details the mapping efficiency for each biological sample. Using MC-Seq sequences mapped to the reference genome with an average mapping efficiency of $90 \%$ across all samples. There were no significant differences in mapping efficiency between tissues and brush swab samples (Fig. 1A). The average difference in mapping efficiency between the paired brush swabs and tissues was minimal, at $-0.567 \%$, in favor of tissue samples, with a range of -1.9 to $1.7 \%$. The majority of methylated C's appeared in a CpG context. We graphed the depth of read for each CpG across all queried CpGs and demonstrated an inflection point at 10x coverage (Fig. 1B). This finding was similar to our previous technical validation study, in which the majority of CpG sites exhibited at least 10x coverage [18]. We therefore applied this cutoff, focusing our analysis on CpG sites with at least 10x coverage. Average number of $\mathrm{CpGs}$ with at least $10 \mathrm{x}$ coverage was 2,716,674 for swab samples and 2,904,261 for tissue samples, with no significant difference between the two sample types, which is in excess of 3-fold greater CpGs interrogated than the most commonly used tool to measure the DNA methylome, the Illumina EPIC array. Figure $1 \mathrm{C}$ indicates the number of $\mathrm{CpGs}$ with at least 10x coverage for each of the 12 individual samples (see also Supplemental Figure 1).

\section{Distribution of methylome regions}

We determined the distribution of $\mathrm{CpG}$ sites profiled by MC-Seq among the CpG sites successfully measured at 10X depth of read or greater overlapping across all 12 samples (3,566,843 CpGs). Figure 1D demonstrates that $36 \%$ were in introns, $26 \%$ were in promoters, $19 \%$ were in exons, and $19 \%$ were in intergenic regions. Overall, MC-Seq provided more robust coverage of functional gene regions in the methylome than typically provided by the EPIC array, detecting ten-fold more $\mathrm{CpG}$ sites in promoter regions and exons than the EPIC array. We determined that 484,697 CpGs from the EPIC array, the majority of which were also found on the $450 \mathrm{~K}(396,409$ $\mathrm{CpG}$ ) were profiled by $\mathrm{MC}$-Seq with at least $10 \mathrm{x}$ coverage. While the breakdown of these CpGs was $33 \%$ intron, $33 \%$ promoter, $15 \%$ exon, and 19\% intergenic, the total number of CpGs in the functional gene regions was proportionally lower owing to the more limited coverage (Fig. 1D).

\section{Correlation between brush swab and tissue biopsies from matched anatomic sites}

Overall, the correlation among CpG site methylation across all samples was high, all exceeding $90 \%$. The average correlation between tissue and brush swabs $(n=12)$ among all $\mathrm{CpG}$ sites shared among the entire sample (cancer + control) $(\mathrm{s}=3,566,843)$ was $93.2 \%(95 \%$ confidence interval: $93.23,93.25 \%$ ). The average correlation between tissue and brush swabs $(n=6)$ among all $\mathrm{CpG}$ sites shared among cancer samples was 91.3\% (95\%

Table 1 Patient demographic characteristics

\begin{tabular}{|c|c|c|c|c|c|c|c|c|c|}
\hline Patient & Age & Sex & Race & Tobacco use, pack years & Alcohol, drinks/wk & Site & TNM & Stage & Grade \\
\hline 1 & 68 & $\mathrm{~F}$ & White & Never & Never & Tongue & T1NOMO & I & Moderate \\
\hline 2 & 68 & M & White & Former, 53 & Former, 24 & Tongue & T4aNOMO & IV & Moderate \\
\hline 3 & 49 & M & White & Current, 72 & Current, 14 & Mandible & T4bN3bM0 & IV & Modera \\
\hline
\end{tabular}


Table 2 Characteristics of genomic DNA used as input for sequencing of tissue and brush swab biopsies

\begin{tabular}{|c|c|c|c|c|c|c|}
\hline Sample & DNA concentration $\mathrm{ng} / \mu \mathrm{l}$ & A260 & A280 & $260 / 280$ & $260 / 230$ & gDNA input, ng \\
\hline 1C swab & 3.96 & 0.01 & -0.004 & -2.99 & -0.18 & 118.8 \\
\hline $1 C$ tissue & 22.00 & 4.45 & 2.210 & 2.01 & 2.18 & 660.0 \\
\hline $1 \mathrm{~N}$ swab & 1.70 & -0.05 & -0.048 & 0.95 & 0.33 & 51.0 \\
\hline $1 \mathrm{~N}$ tissue & 6.24 & 0.44 & 0.220 & 2.01 & 2.75 & 187.2 \\
\hline $2 C$ swab & 4.24 & 0.01 & -0.027 & -0.27 & -0.09 & 127.2 \\
\hline $2 C$ tissue & 11.40 & 1.00 & 0.463 & 2.16 & 2.85 & 342.0 \\
\hline $2 \mathrm{~N}$ swab & 6.32 & 0.07 & 0.014 & 5.14 & -1.45 & 189.6 \\
\hline $2 \mathrm{~N}$ tissue & 8.00 & 0.62 & 0.290 & 2.12 & 3.04 & 240.0 \\
\hline 3C swab & 66.60 & 1.82 & 0.971 & 1.87 & 2.86 & 1998.0 \\
\hline $3 C$ tissue & 21.80 & 2.16 & 1.314 & 1.65 & 0.80 & 654.0 \\
\hline $3 \mathrm{~N}$ swab & 23.60 & 0.45 & 0.217 & 2.06 & 4.70 & 708.0 \\
\hline $3 \mathrm{~N}$ tissue & 8.48 & 0.39 & 0.191 & 2.05 & 5.66 & 254.4 \\
\hline
\end{tabular}

confidence interval: 91.32, 91.35\%). The average correlation between tissue and brush swabs $(n=6)$ among all CpG sites shared among normal samples was $95.1 \%$ (95\% confidence interval: 95.13, 95.14\%). A scatterplot of the CpGs with 10x coverage was generated for the cancer samples and the normal samples separately, demonstrating high concordance between tissue and brush swabs (Fig. 2A and B).

\section{The top methylation features are differentially} methylated between cancer and normal samples, but not between tissues and brush swabs

We focused on the top 1000 most variable methylation features between cancer and normal samples, which would be expected to differ considerably less between tissue and brush swab sampling methods. The $p$-values for each test of difference in CpG methylation by t-test were expressed as $-\log _{10}(p$-value), and averaged 3.67 (i.e., $p=0.00021$ ) between cancer vs. normal. The same CpG sites were not differentially methylated, with an average $-\log _{10}(p$-value $)=0.96$ (i.e., $p=0.11$ ) between tissue vs. brush swabs (Fig. 2C). The results suggest that brush swabs are a clinically viable surrogate for tissue biopsies.

\section{Discussion}

MC-seq is a scalable methylation assay that is currently not widely used in cancer research

EWAS studies in cancer patients have identified interindividual variability in the epigenome, and the recent availability of affordable EWAS technologies have led to a rapid increase in epigenetic biomarker studies aimed at identifying differential methylation features that could be predictive of clinical outcome. The most commonly used platforms are array-based, like the Illumina Human $450 \mathrm{~K}$ and Infinium MethylationEPIC arrays, which provide limited coverage of $\mathrm{CpG}$ sites across the epigenome. Whole genome bisulfite sequencing (WGBS) is the most comprehensive method for epigenome profiling, capturing 28 million CpGs. However, the cost, intensive workflow, and need for high quality and quantity of DNA input significantly limit its clinical translatability, particularly in cancer treatment. MC-Seq has emerged as a promising intermediary between arrays and WGBS, using NGS to capture significantly more CpGs than array-based platforms, while having the advantage of being more high-throughput and affordable than WGBS. We and others have compared $\mathrm{CpG}$ coverage and efficiency of different methylation quantification platforms $[18,24$, 25]. A recent publication from our group has demonstrated that MC-Seq is a more reliable and efficient platform for epigenome profiling than array-based platforms like the EPIC array. When the EPIC array and MC-Seq were compared in peripheral blood mononuclear cell samples, MC-Seq captured significantly more $\mathrm{CpGs}$ in coding regions and $\mathrm{CpG}$ islands than the EPIC array. The EPIC array captured 846,464 CpG sites per sample, whereas MC-Seq captured 3,708,550 CpG sites per sample. Of the $472,540 \mathrm{CpG}$ sites captured by both platforms, there was high correlation $(r=0.98$ 0.99) in methylation status [18]. Moreover, while the EPIC array is enriched for genes with known roles in carcinogenesis, MC-Seq quantifies methylation in a more agnostic manner and profiles 3-4 times more CpGs than the EPIC array, allowing for a higher chance of discovering novel epigenetic modifications in cancer. Furthermore, the coverage areas within each gene were more comprehensive than the EPIC array and other commonly used methylation analysis techniques, like PCR or pyrosequencing. Herein, we demonstrated that MC-Seq captured significantly more CpG sites within functional gene regions, owing to the higher overall profiling capability of this technique. The high throughput capabilities and depth of coverage make MC-Seq an 


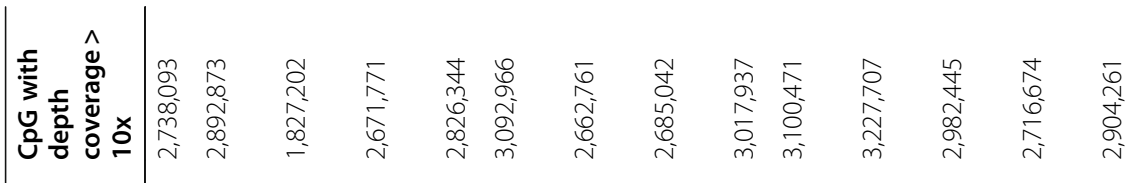

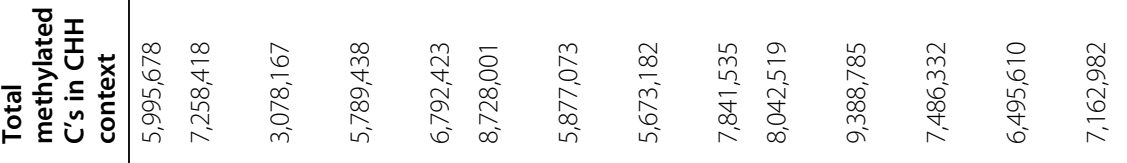

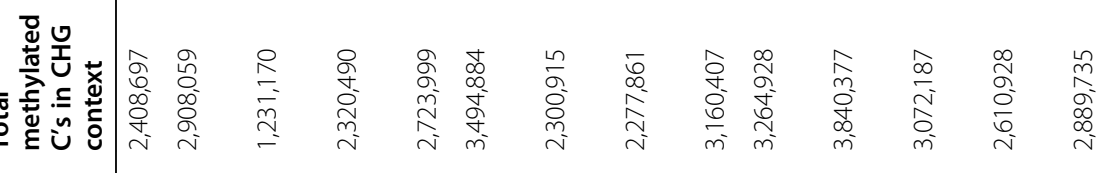

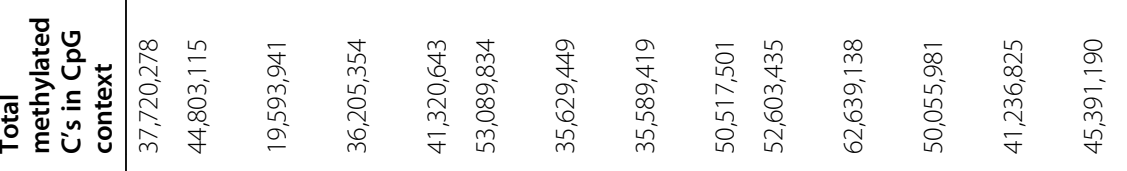

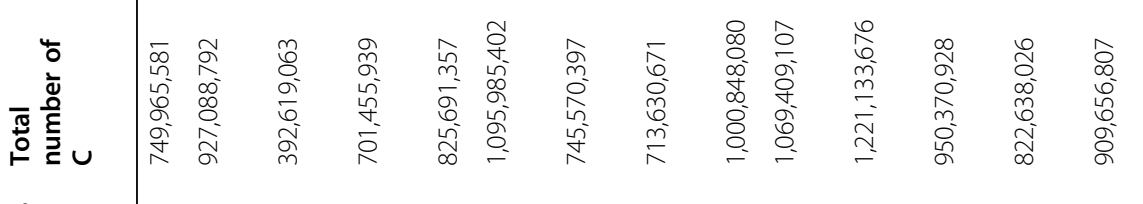

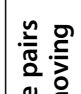

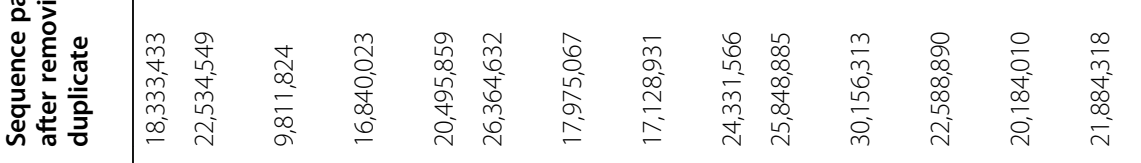
竎 


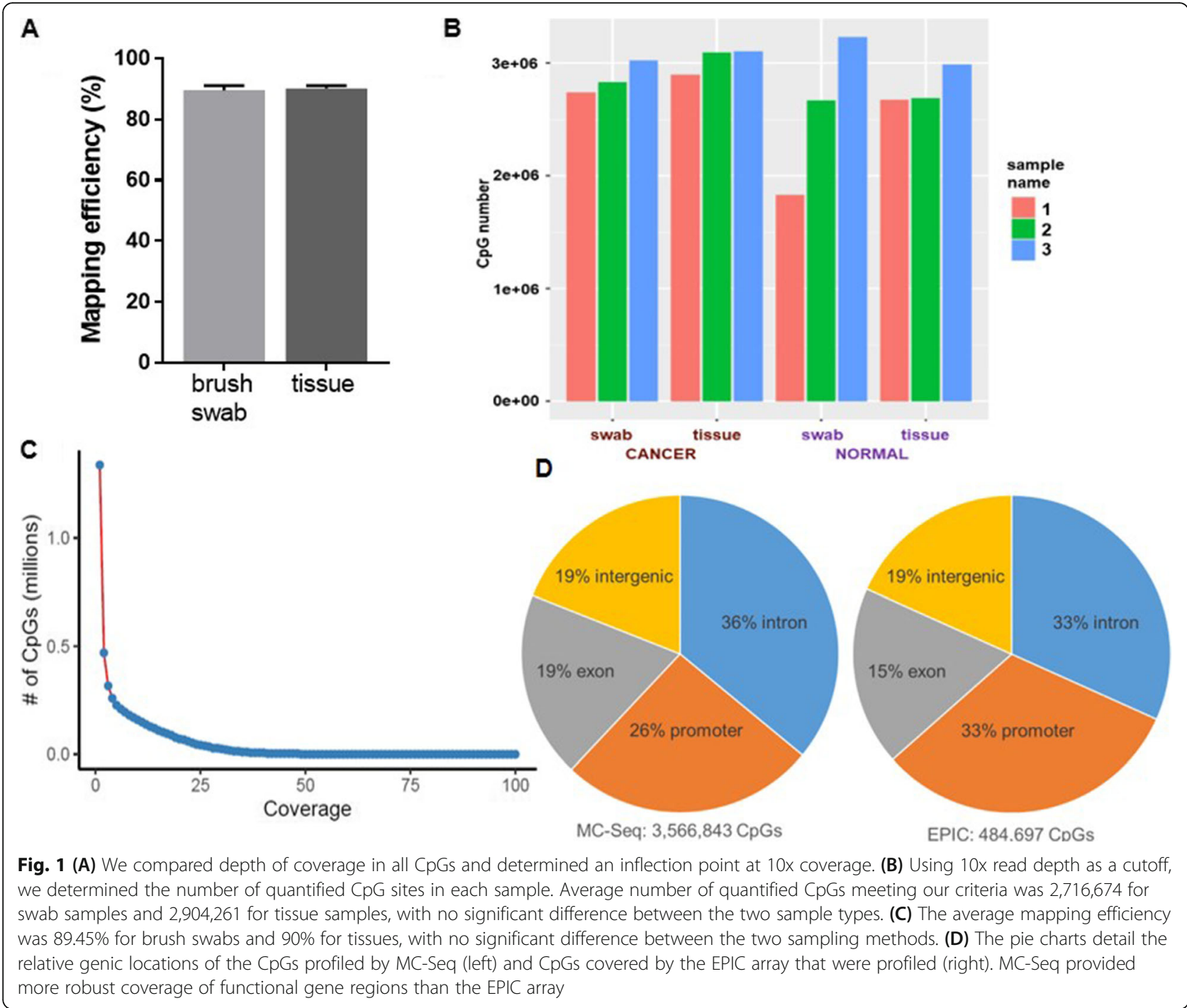

appropriate, CLIA-approvable (Clinical Laboratory Improvement Amendments) platform to be used in a clinical setting.

\section{Oral SCC is an epigenetically-regulated cancer with promising methylation biomarker candidates}

Methylation studies on OSCC patients [6-16] including our own studies $[7,8]$ have demonstrated that methylation is a common event and highlighted specific genes for mechanistic studies. For example, a EWAS using the Illumina Human $450 \mathrm{~K}$ array on 108 head and neck SCC patients of multiple sub-sites including oral cavity identified hypermethylation and inactivation of key tumor suppressor genes [9]. Clinical translation of these methylation biomarker studies has been limited due to: 1) combining OSCC with other head and neck cancer subsites (i.e., oropharynyx, hypopharynx, larynx), which creates a heterogeneous cohort that fails to recognize
OSCC as a distinct clinical disease, and 2) relying solely on array-based platforms, which query a limited number of CpGs. As a result, none of these studies have produced a methylation biomarker with high prognostic performance. We recently used methylation signatures combined with clinicopathologic data to develop a risk score to predict 5-year mortality of early-stage (I/II) OSCC; the risk score accurately predicted mortality with a c-statistic $=0.915$ [5]. The risk score, which we named the REASON score, leveraged the top 12 differentially methylated genes between early-stage OSCC patients who survived vs. died at 5 years after diagnosis. Of note, 11 of the 12 genes had not previously been investigated in OSCC, with our study being the first to correlate differential methylation of these 11 genes with outcomes in OSCC [5].

In addition to being a distinct clinical subsite from other head and neck sites, the oral cavity is an easily 

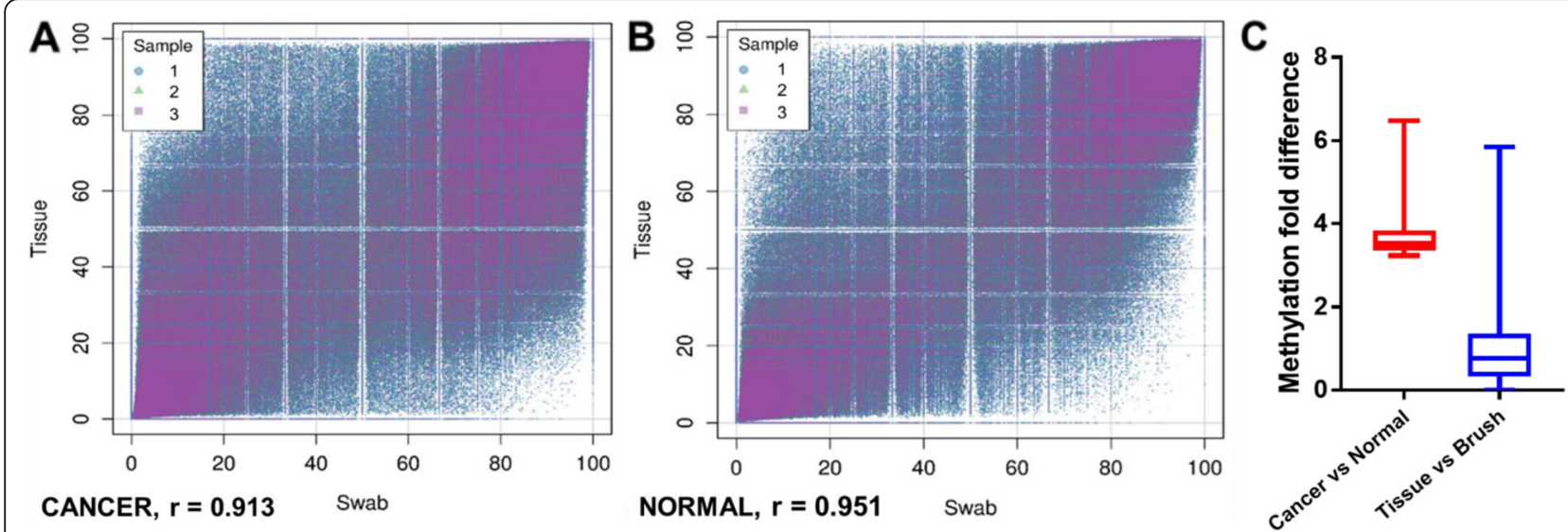

Fig. 2 The scatterplots demonstrate the correlation between tissue and brush swab biopsies for (A) cancer and (B) normal sites of the 3 patients. The correlation values are noted. (C) By focusing on the top 1000 most variable methylation features between cancer and normal samples quantified with MC-Seq, we determined the methylation difference between different sample types, visualized using box plots (median, quartiles, maximum and minimum whiskers). The $p$-values for each test of difference in CpG methylation by t-test were expressed as - $\log _{10}(p$-value), which had a median of 3.67 (i.e., $p=0.00021$ ) between cancer vs. normal. The same CpG sites were not differentially methylated [average - $\log _{10}(p$ value) $=0.96$ (i.e., $p=0.11$ )] between tissue vs. brush swabs, suggesting that brush swabs are a viable surrogate of tissue biopsy

accessible anatomic site for non-invasive biopsy techniques. Clinical translation of a biomarker requires that it can be measured during treatment. Waiting until after tumor removal for the formalin-fixed, paraffinembedded (FFPE) tissues delays potentially necessary treatment. Researchers have used both saliva and brush swabs to noninvasively sample OSCC cells at the time of diagnosis. In our own studies, we have used saliva to identify methylation biomarkers of OSCC. We demonstrated that a multi-gene panel could be constructed using either a methylation array or Methylight, a polymerase chain reaction (PCR) technique $[7,8]$. However, we and others have shown that concordance of methylation between saliva and cancer tissue is highly variable $[26,27]$.

\section{Brush swabs and MC-Seq represent a noninvasive method to quantify methylation biomarkers}

Our approach of using brush swabs and MC-Seq to determine the methylation signature at the time of diagnosis has a high potential for clinical translatability. We demonstrated in this study that brush swab and tissue biopsies from matched sites had highly correlated methylation signatures. Furthermore, the DNA quality and quantity from brush swab samples were adequate to perform MC-Seq. Mapping efficiency was equivalent between tissues and brush swabs. Given the high correlation between the paired tissues and brush swabs, and the satisfactory DNA yield, brush swabs could serve as a clinically robust surrogate to tissue biopsies. One previous study has assessed the reliability of brush swab DNA for MC-Seq compared to the Human $450 \mathrm{~K}$ array [24], drawing similar conclusions to our study [18] that MC-
Seq offered broader coverage of $\mathrm{CpG}$ sites and that sample-based correlation was high $(r=0.98)$ between the two platforms. However, they did not compare brush swab to underlying tissue collection. To our knowledge our study represents the first to directly compare the epigenome-wide signature of matched brush swabs and tissues, with the results having important implications in OSCC biomarker research. Our eventual goal is to apply our methylation risk score (REASON score) to a large cohort of patients, using brush swabs as a noninvasive method to determine methylation signatures for risk stratification.

\section{Conclusions}

Our study establishes a workflow for a large-scale clinical study using brush swab samples and MC-Seq to noninvasively determine the methylation signature of OSCC patients at the time of diagnosis, which could be used to establish risk stratification schemes.

\section{Abbreviations \\ EWAS: epigenome-wide association study; MC-Seq: MethylCap-Seq; OSCC: oral squamous cell carcinoma}

\section{Supplementary Information}

The online version contains supplementary material available at https://doi. org/10.1186/s40364-021-00349-x.

Additional file 1: Supplemental Figure 1. The graphs demonstrate

the M-bias coverage plots for each of the 12 samples

\section{Acknowledgements}

The authors appreciate the support of the Yale Center of Genomic Analysis. We would also like to thank the Women of Head and Neck Reconstructive Surgery (HERS) Consortium. 


\section{Authors' contributions}

CTV and BEA designed the study, wrote the manuscript, and coordinated enrollment of the multiple institutions into the studies. $\mathrm{XZ}, \mathrm{KX}, \mathrm{GY}$ and $\mathrm{KA}$ JFZ performed analysis of the data. CMT, NFC, PCW, KN, SCK, SCL, CTA, SY, ASH, DFR coordinated Institutional Review Board approval, patient enrollment and database creation at their respective sites. CD, JCM, JWS, AG completed the patient databases and performed retrospective chart reviews at their respective institutions. DTV managed the de-identified database. All authors edited the manuscript. The authors read and approved the final manuscript.

\section{Funding}

The study is supported by National Institute on Drug Abuse (R01DA047063 and R01DA047820), National Institute of Dental and Craniofacial Research (K23DE030250) and the Oral Maxillofacial Surgery Foundation.

\section{Availability of data and materials}

All data generated for this study are included in this article.

\section{Declarations}

Ethics approval and consent to participate

Institutional Review Board approval was obtained to collect biological samples and create the de-identified patient databases at each respective institution.

\section{Consent for publication}

Not applicable.

\section{Competing interests}

Not applicable.

\section{Author details}

'Department of Oral and Maxillofacial Surgery, Loma Linda University School of Dentistry, Loma Linda, CA, USA. ${ }^{2}$ Department of Psychiatry, Yale School of Medicine, New Haven, CT, USA. ${ }^{3}$ VA Connecticut Healthcare System, West Haven, CT, USA. ${ }^{4}$ New York University Rory Meyers College of Nursing, New York, NY, USA. ${ }^{5}$ Department of Oral and Maxillofacial Surgery, New York University College of Dentistry, New York, NY, USA. ${ }^{6}$ Bluestone Center for Clinical Research, New York University College of Dentistry, New York, NY, USA. 'Department of Otolaryngology, University of Alabama at Birmingham, Birmingham, AL, USA. ${ }^{8}$ Department of Oral and Maxillofacial Surgery, University of Illinois Chicago, College of Dentistry, Chicago, IL, USA. ${ }^{9}$ Department of Otolaryngology, Loma Linda University School of Medicine, Loma Linda, CA, USA. ${ }^{10}$ Section on Translational Tumor Immunology, National Institute on Deafness and Other Communication Disorders (NIDCD), National Institutes of Health (NIH), Bethesda, MD, USA. ${ }^{11}$ Department of Oral, Head and Neck Oncology and Microvascular Reconstructive Surgery, School of Dentistry, University of Texas Health Science Center at Houston, Houston, TX, USA. ${ }^{12}$ Rocky Vista University, Ivins, UT, USA. ${ }^{13}$ Department of Otolaryngology, Rutgers New Jersey Medical School, Newark, NJ, USA. ${ }^{14}$ Department of Pathology, University of Alabama at Birmingham, Birmingham, AL, USA. ${ }^{15}$ Department of Basic Sciences, Loma Linda University, School of Medicine, Loma Linda, CA, USA.

Received: 7 July 2021 Accepted: 28 October 2021

Published online: 20 December 2021

\section{References}

1. Gulland A. Oral cancer rates rise by two thirds. BMJ. 2016;355:i6369. https:// doi.org/10.1136/bmj.i6369.

2. Tota JE, Anderson WF, Coffey C, Califano J, Cozen W, Ferris RL, et al. Rising incidence of oral tongue cancer among white men and women in the United States, 1973-2012. Oral Oncol. 2017;67:146-52. https://doi.org/10.101 6/j.oraloncology.2017.02.019.

3. Kim YJ, Kim JH. Increasing incidence and improving survival of oral tongue squamous cell carcinoma. Sci Rep. 2020;10(1):7877. https://doi.org/10.1038/ s41598-020-64748-0

4. Yoon AJ, Wang S, Kutler DI, Carvajal RD, Philipone E, Wang T, et al. MicroRNA-based risk scoring system to identify early-stage oral squamous cell carcinoma patients at high-risk for cancer-specific mortality. Head Neck. 2020:42(8):1699-712. https://doi.org/10.1002/hed.26089.

5. Viet CT, Yu G, Asam K, Thomas CM, Yoon AJ, Wongworawat YC, et al. The REASON Score: An Epigenetic and Clinicopathologic Score to Predict Risk of Poor Survival in Patients with Early Stage Oral Squamous Cell Carcinoma. Biomark Res. 2021;9(1):9. https://doi.org/10.1186/s40364-021-00292-x.

6. Poage GM, Houseman EA, Christensen BC, Butler RA, Avissar-Whiting M, McClean MD, et al. Global hypomethylation identifies loci targeted for hypermethylation in head and neck cancer. Clin Cancer Res. 2011;17(11): 3579-89. https://doi.org/10.1158/1078-0432.CCR-11-0044.

7. Viet $\mathrm{CT}$, Jordan RC, Schmidt BL. DNA promoter hypermethylation in saliva for the early diagnosis of oral cancer. J Calif Dent Assoc. 2007;35(12):844-9.

8. Viet CT, Schmidt BL. Methylation array analysis of preoperative and postoperative saliva DNA in oral cancer patients. Cancer Epidemiol Biomark Prev. 2008;17(12):3603-11. https://doi.org/10.1158/1055-9965.EPI-08-0507.

9. Guerrero-Preston R, Michailidi C, Marchionni L, Pickering CR, Frederick MJ, Myers JN, et al. Key tumor suppressor genes inactivated by "greater promoter" methylation and somatic mutations in head and neck cancer. Epigenetics. 2014;9(7):1031-46. https://doi.org/10.4161/epi.29025.

10. Ha PK, Califano JA. Promoter methylation and inactivation of tumoursuppressor genes in oral squamous-cell carcinoma. Lancet Oncol. 2006;7(1): 77-82. https://doi.org/10.1016/S1470-2045(05)70540-4.

11. Huang MJ, Yeh KT, Shih HC, Wang YF, Lin TH, Chang JY, et al. The correlation between $\mathrm{CpG}$ methylation and protein expression of P16 in oral squamous cell carcinomas. Int J Mol Med. 2002;10(5):551-4.

12. Shaw RJ, Hall GL, Woolgar JA, Lowe D, Rogers SN, Field JK, et al. Quantitative methylation analysis of resection margins and lymph nodes in oral squamous cell carcinoma. Br J Oral Maxillofac Surg. 2007:45(8):617-22. https://doi.org/10.1016/j.bjoms.2007.04.015.

13. Shaw RJ, Liloglou T, Rogers SN, Brown JS, Vaughan ED, Lowe D, et al. Promoter methylation of P16, RARbeta, E-cadherin, cyclin A1 and cytoglobin in oral cancer: quantitative evaluation using pyrosequencing. $\mathrm{Br} J$ Cancer. 2006;94(4):561-8. https://doi.org/10.1038/sj.bjc.6602972

14. Smiraglia DJ, Smith LT, Lang JC, Rush LJ, Dai Z, Schuller DE, et al. Differentia targets of $\mathrm{CpG}$ island hypermethylation in primary and metastatic head and neck squamous cell carcinoma (HNSCC). J Med Genet. 2003:40(1):25-33. https://doi.org/10.1136/jmg.40.1.25

15. Yeh KT, Shih MC, Lin TH, Chen JC, Chang JY, Kao CF, et al. The correlation between $\mathrm{CpG}$ methylation on promoter and protein expression of E-cadherin in oral squamous cell carcinoma. Anticancer Res. 2002;22(6C):3971-5.

16. Li YF, Hsiao YH, Lai YH, Chen YC, Chen YJ, Chou JL, et al. DNA methylation profiles and biomarkers of oral squamous cell carcinoma. Epigenetics. 2015; 10(3):229-36. https://doi.org/10.1080/15592294.2015.1006506.

17. Eljabo N, Nikolic N, Carkic J, Jelovac D, Lazarevic M, Tanic N, et al. Genetic and epigenetic alterations in the tumour, tumour margins, and normal buccal mucosa of patients with oral cancer. Int J Oral Maxillofac Surg. 2018; 47(8):976-82. https://doi.org/10.1016/j.ijom.2018.01.020.

18. Shu C, Zhang X, Aouizerat BE, Xu K. Comparison of methylation capture sequencing and Infinium MethylationEPIC array in peripheral blood mononuclear cells. Epigenetics Chromatin. 2020;13(1):51. https://doi.org/1 0.1186/s13072-020-00372-6.

19. Gissi DB, Morandi L, Colella G, De Luca R, Campisi G, Mauceri R, et al. Clinical validation of 13-gene DNA methylation analysis in oral brushing samples for detection of oral carcinoma: Italian multicenter study. Head Neck. 2021:43(5):1563-73. https://doi.org/10.1002/hed.26624.

20. Morandi L, Gissi D, Tarsitano A, Asioli S, Gabusi A, Marchetti C, et al. CpG location and methylation level are crucial factors for the early detection of oral squamous cell carcinoma in brushing samples using bisulfite sequencing of a 13-gene panel. Clin Epigenetics. 2017;9(1):85. https://doi. org/10.1186/s13148-017-0386-7.

21. Gissi DB, Fabbri VP, Gabusi A, Lenzi J, Morandi L, Melotti S, et al. PreOperative Evaluation of DNA Methylation Profile in Oral Squamous Cell Carcinoma Can Predict Tumor Aggressive Potential. Int J Mol Sci. 2020; 21(18):21. https://doi.org/10.3390/ijms21186691.

22. Lydiatt WM, Patel SG, O'Sullivan B, Brandwein MS, Ridge JA, Migliacci JC, et al. Head and neck cancers-major changes in the American joint committee on cancer eighth edition cancer staging manual. CA Cancer J Clin. 2017:67(2):122-37. https://doi.org/10.3322/caac.21389.

23. Taylor JM, Ankerst DP, Andridge RR. Validation of biomarker-based risk prediction models. Clin Cancer Res. 2008;14(19):5977-83. https://doi.org/1 0.1158/1078-0432.CCR-07-4534. 
24. Teh AL, Pan H, Lin X, Lim Yl, Patro CP, Cheong CY, et al. Comparison of methyl-capture sequencing vs. Infinium $450 \mathrm{~K}$ methylation array for methylome analysis in clinical samples. Epigenetics. 2016;11(1):36-48. https://doi.org/10.1080/15592294.2015.1132136.

25. Heiss JA, Brennan KJ, Baccarelli AA, Tellez-Rojo MM, Estrada-Gutierrez G, Wright RO, et al. Battle of epigenetic proportions: comparing Illumina's EPIC methylation microarrays and TruSeq targeted bisulfite sequencing. Epigenetics. 2020;15(1-2):174-82. https://doi.org/10.1080/15592294.2019.1 656159.

26. Arantes L, De Carvalho AC, Melendez ME, Lopes Carvalho A. Serum, plasma and saliva biomarkers for head and neck cancer. Expert Rev Mol Diagn. 2018;18(1):85-112. https://doi.org/10.1080/14737159.2017.1404906.

27. Nishitani S, Parets SE, Haas BW, Smith AK. DNA methylation analysis from saliva samples for epidemiological studies. Epigenetics. 2018;13(4):352-62. https://doi.org/10.1080/15592294.2018.1461295.

\section{Publisher's Note}

Springer Nature remains neutral with regard to jurisdictional claims in published maps and institutional affiliations.

Ready to submit your research? Choose BMC and benefit from:

- fast, convenient online submission

- thorough peer review by experienced researchers in your field

- rapid publication on acceptance

- support for research data, including large and complex data types

- gold Open Access which fosters wider collaboration and increased citations

- maximum visibility for your research: over $100 \mathrm{M}$ website views per year

At BMC, research is always in progress.

Learn more biomedcentral.com/submissions 